\title{
Site-specific Modular Design Optimization for High-rise Residential Buildings
}

\author{
Vincent J.L. Gan ${ }^{1}$, K.T. Tse ${ }^{1}$, Jack C.P. Cheng ${ }^{1}$, Irene M.C. Lo ${ }^{1}$, and C.M. Chan ${ }^{1 *}$ \\ ${ }^{1}$ Research Assistant Professor, Dept. of Civil and Environmental Engineering, \\ The Hong Kong University of Science and Technology (HKUST) \\ ${ }^{2}$ Associate Professor, Dept. of Civil and Environmental Engineering, HKUST \\ ${ }^{3}$ Associate Professor, Dept. of Civil and Environmental Engineering, HKUST \\ ${ }^{4}$ Chair Professor, Dept. of Civil and Environmental Engineering, \\ Institute for Advanced Study (IAS) Senior Fellow, HKUST \\ ${ }^{5}$ Professor, Dept. of Civil and Environmental Engineering, HKUST \\ ${ }^{*}$ Corresponding author's e-mail: cecmchan@ust.hk
}

\begin{abstract}
Modular design refers to a design approach whereby customized modules or components are assembled to form the layout plan of a building. Previous researches have attempted to optimize the layout plan design of low-rise houses for maximizing the natural daylighting, ventilation performance, and energy efficiency. Engineers have also studied the modular design of high-rise residential buildings to meet site constraints and to optimize site development potentials. However, the previous studies on modular building design were based on empirical trial-anderror approaches, efficient methods for identifying the optimal combination of different modules and components were still lacking in literature. Therefore, this study attempts to develop an innovative approach for optimizing the modular design of high-rise residential buildings, with the aim of maximizing the building energy performance while fulfilling the site constraints and design code requirements. The design optimization problem, including the design variables and objective functions, is properly formulated to guarantee the quality of final optimized deign. Provided a set of well-defined modules and components, evolutionary genetic algorithm (GA) is then utilized for the wide-ranging exploration of the building layout plans, taking into consideration the site conditions and building design requirements. A computer program is developed, coupling the GA optimization and energy modeling, to systematically evaluate the candidate layout plans. The energy simulation results are subsequently used to guide the GA towards finding the optimal design solution. The proposed optimization method is utilized to generate the optimal layout design for a 40 -story high-rise residential building, using a set of predefined modular flat units. The optimal design maximizes the use of natural ventilation and daylighting to save $30-40 \%$ of the energy consumption without compromising the site constraints and design requirements. The findings of this study serve as the decision support basis to enhance modular design of high-rise residential buildings (such as energy conservation in this study), thereby improving the sustainability and cost-effectiveness of the built environment.
\end{abstract}

\section{KEYWORDS}

Design optimization; Energy efficiency; Genetic algorithm; High-rise residential building; Modular design; Sustainability 


\section{INTRODUCTION}

Modular design is an approach whereby discrete and well-defined modules or components are strategically integrated together to form the layout plan of a building. Followed by building design, the volumetric modules and components are prefabricated in factories and transported to construction sites for installation. Modular design approach has large potentials to enhance the traditional building layout plan design and the sustainability of built environment. Bojic et al. (2001) compared the energy performance of two standardized flat layout designs (two-bedroom and three-bedroom flats) for residential buildings with different thermal insulation materials and construction methods. Some researchers have also attempted to optimize the building layout plan and to explore the best physical arrangement of all spaces within a building to achieve the best overall performance (Bao et al., 2013). In addition, Wang (2005) and Wang et al. (2006) applied an evolutionary optimization method based on the genetic algorithm (GA) to identify the optimal building layout plan of a multi-story building, with the aim of minimizing the life cycle energy use in buildings. Flager et al. (2009) optimized the dimensions and structural components of an individual room by integrating energy simulation with GA multi-objective optimization for lower energy usage and reduced structural cost. Moreover, Merrell (2010) optimized the layout plan of living rooms, bedrooms, kitchen etc. in a low-rise house by a stochastic optimization method using a Bayesain network trained on real-world data.

Most of the previous studies related to the standardized flat design or building layout plan focused on low-rise or multi-story residential buildings with a generally regular shape. There have been some studies emphasizing the modular design for high-rise residential buildings, taking into consideration tenants' need, daylighting requirement, and buildability (Cheng, 2011). Hong Kong Housing Authority (2012) also presented a modular flat design approach that can be used to cater for the site condition variance, maximize the land resource, and enhance the building efficiency. In addition, Hong Kong Institute of Architects (2012) discussed the sitespecific modular flat design approach regarding its improvements to the quality, built environment, and cost-effectiveness of high-rise residential buildings. However, the previous studies on modular high-rise residential buildings rely heavily on empirical methods and trialand-error processes to improve the building efficiency, robust methods for the design optimization of high-rise residential buildings using modular units and components were still lacking in literature.

Therefore, this study attempts to develop a novel approach for optimizing the modular design of high-rise residential buildings, with the aim of maximizing the building sustainability (i.e., energy performance) while fulfilling the site constraints and design code requirements. The optimization problem is first mathematically formulated, considering design variables and constraints. Based on the mathematical formulation, the evolutionary GA is utilized for the wideranging exploration of the building layout plans, using a set of customized modular flat units and components. Site conditions and building design requirements are considered in the design optimization process. Each building layout plan formed by the modular units is evaluated in energy modeling program, the results of which are then used to guide the GA towards finding the optimal design solution. A customized program is developed to couple the GA optimization with energy simulation in order to systematically explore the design space and to identify the most efficient and sustainable modular building design. 


\section{METHODOLOGY}

The proposed method aims to optimize high-rise residential building design using modular units and components for maximizing the energy efficiency and sustainability of buildings. As shown in Figure 1, the proposed method starts by defining a set of modular units and components with standardized configurations/dimensions. The standardized modules are then combined to form different wings, which are subsequently utilized to create the entire building layout plan in consideration of the site condition. Generic algorithm (GA) is used to generate and optimize the building layout plans, taking into consideration the energy efficiency and sustainability criteria. GA is an efficient search heuristic that models the natural selection process and uses the fittest individuals to reproduce and search for the new generation of qualified design solutions (Wetter and Wright, 2004). The final optimized layout plan is used to generate the whole building model. Details about the design optimization are described in the following sections.

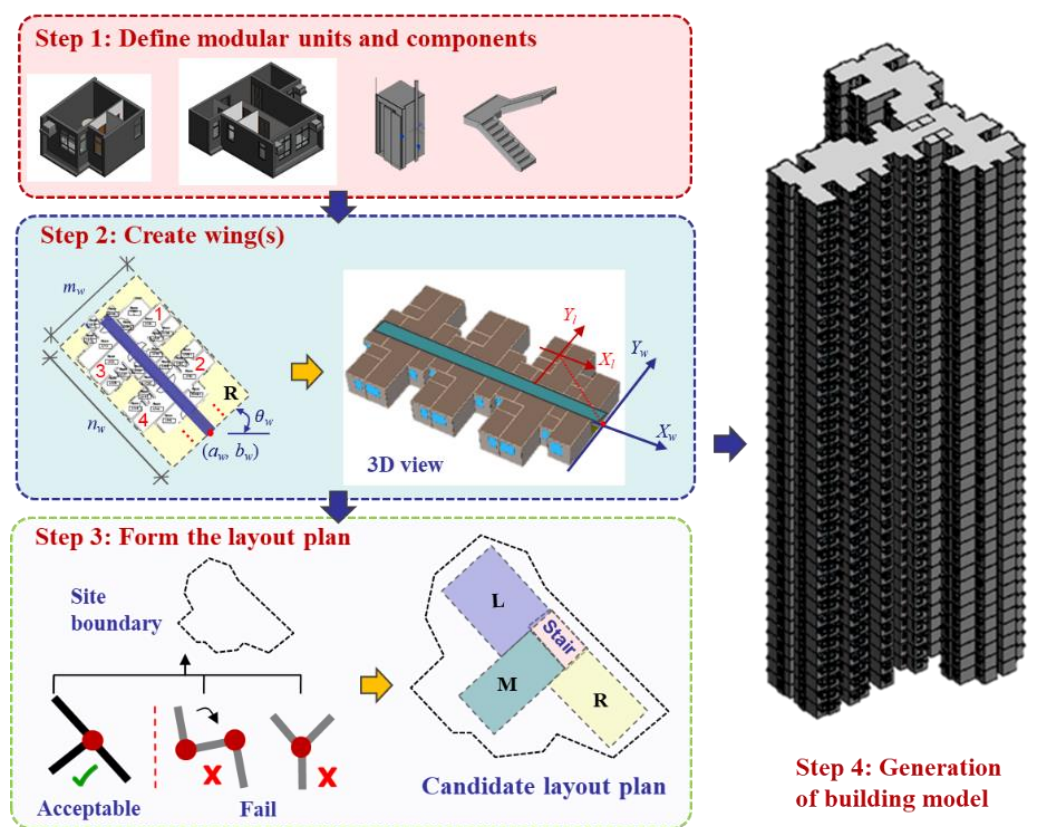

Figure 1. Design workflow for modular high-rise residential buildings.

\section{Problem Formulation}

As proper formulation of the design optimization problem is critical for the final optimized design, the first step is to properly formulate the building layout optimization, including the design variables, objective functions, and constraints. The energy performance of each modular building design is evaluated using an objective function that emphasizes the major energyconsuming activities during building operation such as air-conditioning and lighting. Eq. (1) refers to the objective function, which aims to minimize the energy consumption.

subject to:

$$
\text { Minimize } \operatorname{TEC}(\Gamma)=\sum_{w=1}^{W} \sum_{l=1}^{L_{w}}\left[\sum_{t=1}^{T} P_{w}^{l}(t)+\sum_{t=1}^{T} Q_{w}^{l}(t)\right]
$$

$$
F_{w}^{l} \in \bigcup B \quad \Leftrightarrow \quad \exists F_{w}^{l} \in \Gamma
$$


MOC SUMMIT / MAY 2019

$$
\begin{aligned}
& \sum_{w=1}^{W} \sum_{l=1}^{L_{w}} A_{w}^{l} \geq C_{\varepsilon} \\
& \sum_{w=1}^{W} \sum_{l=1}^{L_{w}} \vartheta_{w}^{l} \geq C_{\delta}
\end{aligned}
$$

in which $\operatorname{TEC}(\Gamma)$ stands for the total energy consumption associated with air-conditioning and lighting $(\mathrm{kWh}), \Gamma$ represents a specific layout plan, $w$ refers to a wing in the layout plan $\Gamma, l$ represents a specific type of flat in wing $w, t$ refers to a particular time step (hour). $P_{w}^{l}(t)$ represents the lighting electricity consumption for time $t(\mathrm{kWh})$, and $Q_{w}^{l}(t)$ stands for the electricity consumption for air-conditioning at time $t$ for flat $l$ in wing $w(\mathrm{kWh}) . P_{w}^{l}(t)$ and $Q_{w}^{l}(t)$ are obtained from the building energy simulation. The operating status of the lighting depends on the indoor illuminance that is incident on reference surface and the minimum illuminance per unit surface area required to maintain a comfortable visual environment. The lighting is turned on whenever the indoor illuminance level is lower than the minimum requirement; otherwise, lighting is turned off for energy-saving purposes. Similarly, the air-conditioning is turned on whenever the indoor temperature is higher than a pre-defined set-point temperature. The electricity use for air-conditioning is calculated, considering the thermal load and the coefficient of performance for the air-conditioning system. The thermal load is calculated as the total amount of heat exchange between the indoor and outdoor environment, considering four typical components: (i) heat gain or loss by conduction through opaque and transparent surfaces; (ii) heat gain of solar radiation; (iii) internal heat loads from human bodies, household equipment, and lighting; and (iv) heat gain or loss due to infiltration and/or ventilation air.

Eqs. (2)-(4) represent the design constraints for generating layout plans. Eq. (2) shows that any flat $F_{w}^{l}$ belonging to the layout plan $\Gamma$ should be located inside the site boundary denoted by $\cup B$. Eq. (3) specifies that the total area of all flats in a layout plan $\left(\sum_{w=1}^{W} \sum_{l=1}^{L_{w}} A_{w}^{l}\right)$ must be more than or equal to the target floor area $C_{\varepsilon}$. Eq. (4) denotes that the expected number of occupants $\left(\sum_{w=1}^{W} \sum_{l=1}^{L_{w}} \vartheta_{w}^{l}\right)$ should be more than or equal to the target number of residents $C_{\delta}$.

\section{Optimization by Genetic Algorithm}

In the GA optimization, each modular building layout plan is represented by a bit string, in which every digit interprets a specific characteristic of the layout plan. As shown in Figure 2, the bit string consists of two major parts, the first of which (3-7 digits) describes the geometrical combination of wings and the building orientation, whereas the second part (max 28 digits) interprets the detailed arrangements of modular units (such as the modular flats). Provided a set of pre-defined modular units and components, the GA first randomly combine modules and produces an initial population of modular building layout plans (called parents). Each parent layout is then altered by crossover and mutation functions in the GA to form a new generation of building layout designs (called offspring). For example, a pair of parent layout plans are randomly selected using the stochastic uniform method and the crossover function exchanges a certain part of the bit strings to alter the combination of modular units and components. Given the new generation of modular building layout plans, the GA produces the corresponding energy models in order to quantify the amount of energy consumption from air-conditioning and 
lighting. The energy performance of each layout plan is subsequently evaluated in energy simulation program and then used to guide the GA towards finding the optimal design option with the least energy consumption. The offspring solutions that meet all the design constraints while showing reduced energy consumption are selected as the new generation of parent solutions in the next iteration of the algorithm. The GA optimization terminates if the maximum number of generations has been reached, or a satisfactory energy performance has been achieved (e.g., no further improvement after certain iteration). The optimal modular building design with the least energy consumption can then be identified.

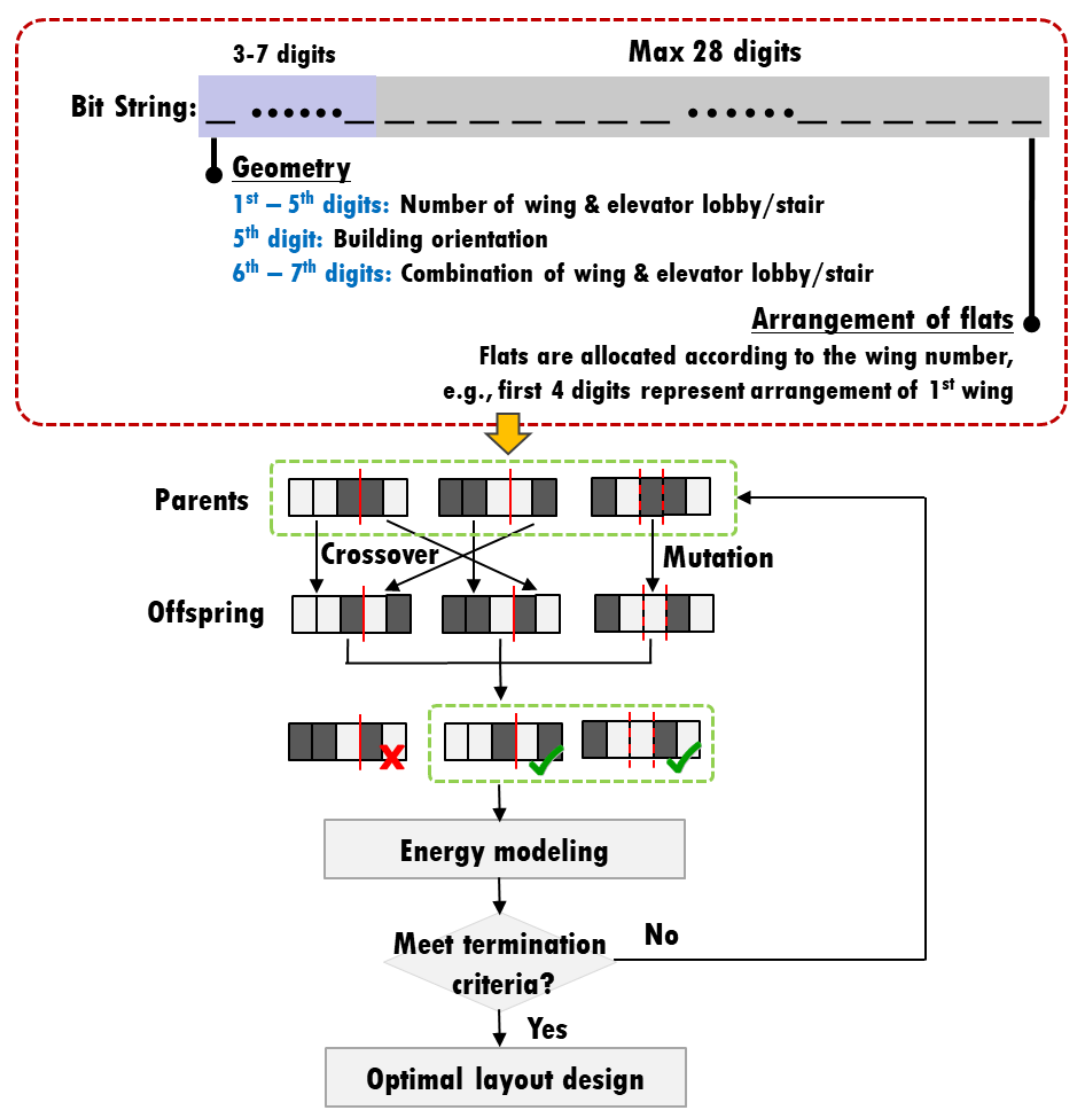

Figure 2. Proposed GA-based optimization approach.

\section{Case Study}

A customized program is developed to implement the layout design optimization for modular high-rise residential buildings. The energy simulation is conducted using eQUEST (2016), which is a widely recognized energy modeling tool by green building standards. The customized program couples the GA optimization with the energy simulation in order to automatically explore the most energy-efficient layout plan for high-rise residential buildings. A case study is conducted to illustrate the proposed optimization approach.

A 32-story T-shape public housing in Hong Kong is selected as the original design for applying the proposed approach. As shown in Figure 3, the reference building consists of 18 modular flats for each floor, including eight 1/2 Person flats, eight 1-Bedroom flats, and two 2-Bedroom flats. Assuming a maximum of two persons living in 1/2 Person flats, three persons in 1-Bedroom flats, and four persons in 2-Bedroom flats, the total number of occupants for each floor is estimated to 
be 48. The standardized flats form three separate wings, which are then connected by an elevator lobby in the middle of the building. The building is a concrete structure, with shear walls constructed from C40 concrete and floor slabs from C30 concrete. Figure 4 shows three types of modular flats that are used in the building layout plan optimization. The optimized design follows specifications and site conditions for the original design. For the ease of comparison, the energy modeling includes only the energy use for air-conditioning over the cooling season from April to October.

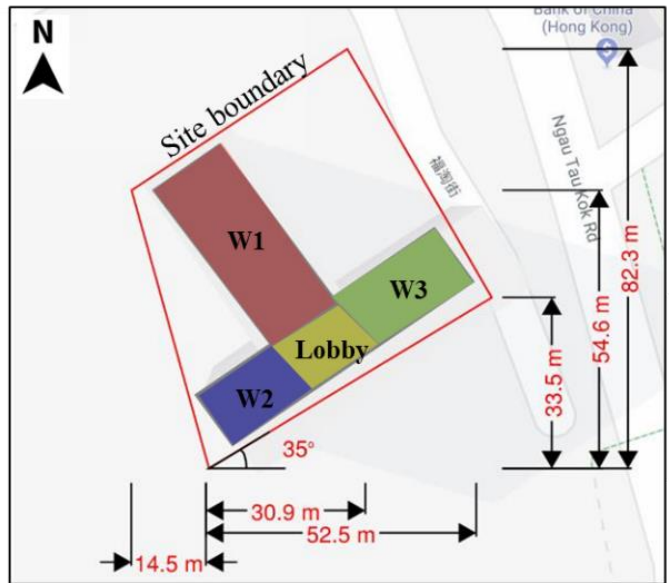

(a)

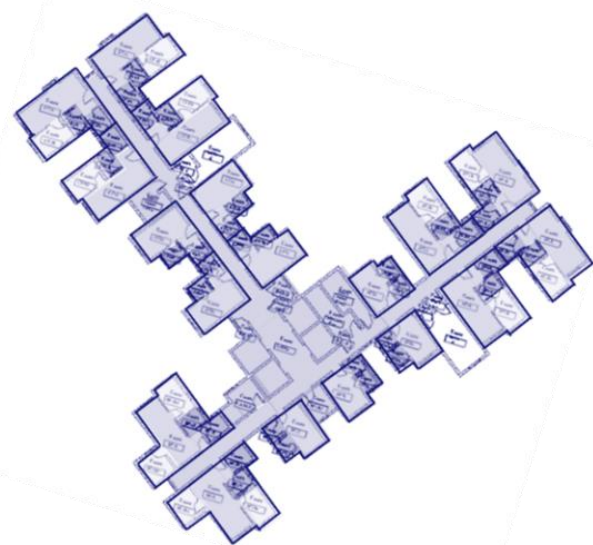

(b)

Figure 3. (a) Overview and (b) floor plan for the reference building.

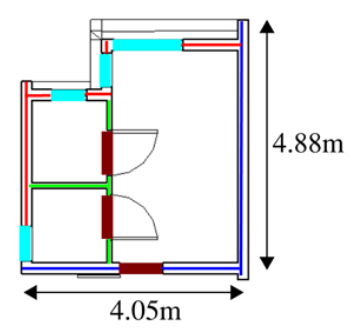

1/2P Flat

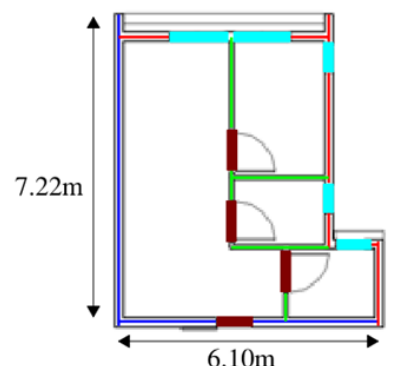

1 Bedroom Flat

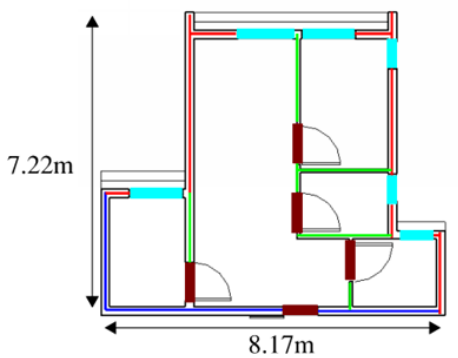

2 Bedroom Flat

Figure 4. Modular flat units used in the optimization.

\section{RESULTS AND DISCUSSION}

The annual electricity consumptions associated with air-conditioning for the original and optimized designs are presented in this section.

\section{Energy Performance for Original and Optimized Designs}

Table 1 compares the annual electricity consumption for air-conditioning associated with the original and optimized designs. For the original design, the electricity demand for airconditioning of all the flats in one floor is $24.1 \mathrm{MWh}$, and the average electricity use per floor area is around $56.2 \mathrm{kWh} / \mathrm{m}^{2}$. The total electricity consumption for the optimized design is 18.6 MWh and the per-area electricity consumption $43.7 \mathrm{kWh} / \mathrm{m}^{2}$, which are $22 \%$ less than that of the 
original design. The proposed optimization method can substantially enhance the energy performance and sustainability of high-rise residential buildings.

Table 1. Annual electricity consumption of air-conditioning for original and optimized designs.

\begin{tabular}{lcc}
\hline & Original & Optimized \\
\hline $\begin{array}{l}\text { Annual electricity consumption } \\
\text { for all flats in one floor }(\mathrm{MWh})\end{array}$ & 24.1 & 18.6 \\
$\begin{array}{l}\text { Overall area of all flats }\left(\mathrm{m}^{2}\right) \\
\begin{array}{l}\text { Annual electricity consumption } \\
\text { per floor area }\left(\mathrm{kWh} / \mathrm{m}^{2}\right)\end{array}\end{array}$ & 429 & 426 \\
\hline
\end{tabular}

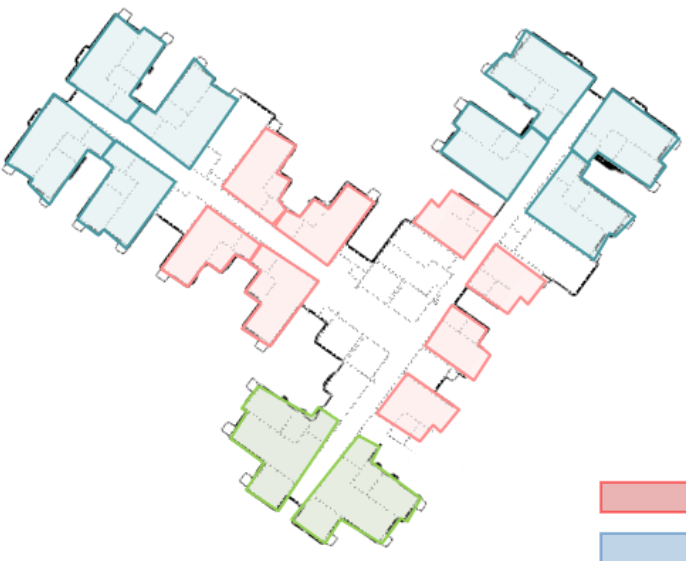

(a)

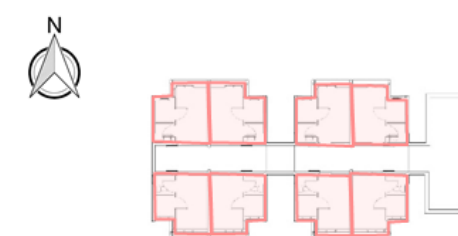

$1 / 2$ Person flat

1-Bedroom flat

2-Bedroom flat

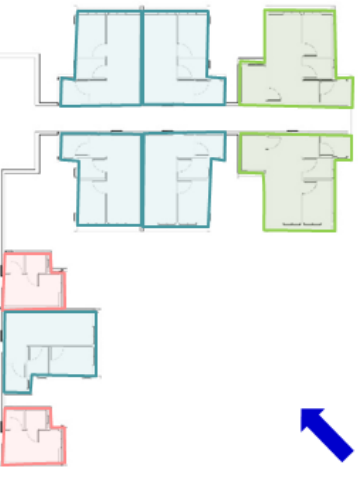

Prevailing wind

(b) direction

Figure 5. Position of modular flats for (a) original and (b) optimized designs.

Figure 5 compares the layout plans for the original and optimized designs. In most cases, the layout design in sub-tropical cities like Hong Kong emphasize air-conditioning because the hot and humid weather will increase the need for air-conditioning. Solar radiation and natural ventilation are the two most critical thermal load components for determining the electricity consumption of air-conditioning. Therefore, it is important to optimize the combination of modular flats and the layout plan for minimizing heat gain of solar radiation while maximizing wind capture for natural ventilation. In Hong Kong, the prevailing wind direction is southeast and there are more periods in the year that wind carries cool air from east and southeast directions (see Figure 5). The proposed GA optimization method strategically allocates the large-sized modular flats at the southeast corner, since the large-sized modular flats (such as 1Bedroom and 2-Bedroom flats) are more energy demanding and difficult to be cooled. The prevailing wind from east and southeast directions provide better natural ventilation to cool the indoor air temperature in large-sized flats, thereby saving the electricity consumption for airconditioning. In contrast, small-sized modular flats (i.e., 1/2 Person flat) are less energydemanding and easier to be cooled, therefore they are positioned at the west wing where winddriven natural ventilation is relatively less efficient. Although smaller-sized modular flats have less wind-driven natural ventilation, the indoor and outdoor temperature difference (also referred to as the buoyancy effect) can also provide airflow which is sufficient to create a pleasant indoor thermal environment for small-sized modular flats. The results indicate that allocating large 
modular flats to face the prevailing wind directions is an energy-efficient practice and help control the indoor environment. In some cases, modular flats facing prevailing wind directions may maximize natural ventilation, but it may also increase the heat gain from solar radiation. In such circumstances, the proposed optimization approach need to analyze the combined effects of solar radiation and natural ventilation, as well to generate the optimized modular design.

\section{CONCLUSION}

This paper presents an evolutionary GA optimization approach to identify the optimal layout plan for minimizing the energy consumption of high-rise residential buildings, considering various design constraints. A customized program is developed to systematically explore the optimal modular design of a 40-story high-rise residential building, using a set of well-defined modular flat units. The optimization results provides insights regarding the critical parameters relevant to the ultimate energy performance of modular high-rise residential buildings, and serves as a decision support tool for enhancing the building sustainability. The actual layout plan may differ for regions with different weathers, site conditions, materials, and occupancy patterns, but the same optimization method can be used to find the corresponding optimal design options.

\section{REFERENCES}

Bao, F., Yan, D.-M., Mitra, N.J., Wonka, P., (2013). "Generating and exploring good building layouts." ACM Transactions on Graphics (TOG) 32(4), 122.

Bojic, M., Yik, F., Sat, P., (2001). "Influence of thermal insulation position in building envelope on the space cooling of high-rise residential buildings in Hong Kong." Energy and Buildings 33(6), 569-581.

Cheng, I., (2011). "Affordable social housing: modular flat design for mass customization in public rental housing in Hong Kong," Proceedings of SB11 Helsinki World Sustainable Building Conference. Helsinki, Finland.

eQUEST 3.65, (2016). "eQUEST: the QUick Energy Simulation Tool." James J. Hirsch \& Associates, US.

Flager, F., Welle, N., Bansal, P., Soremekun, G., Haymaker, J., (2009). "Multidisciplinary process integration and design optimization of a classroom building." Journal of Information Technology in Construction 14, 595-612.

Hong Kong Housing Authority, (2012). "Modular Flat Design for Public Housing Development of the Hong Kong Housing Authority." Hong Kong Housing Authority, Hong Kong.

Hong Kong Institute of Architects, (2012). "Public Housing - Improvement in Quality of Life." Hong Kong Institute of Architects, Hong Kong.

Merrell, P., Schkufza, E., Koltun, V., (2010). "Computer-generated residential building layouts." ACM Transactions on Graphics (TOG) 29(6), 181.

Wang, W., Rivard, H., Zmeureanu, R., (2006). "Floor shape optimization for green building design." Advanced Engineering Informatics 20(4), 363-378.

Wang, W., Zmeureanu, R., Rivard, H., (2005). "Applying multi-objective genetic algorithms in green building design optimization." Building and Environment 40(11), 1512-1525.

Wetter, M., Wright, J., (2004). "A comparison of deterministic and probabilistic optimization algorithms for nonsmooth simulation-based optimization." Building and Environment 39(8), 989-999. 